focal, absence and atonic seizures evolve between 1 and 4 years, and are accompanied by slow development and regression. Neurologic abnormalities include spasticity and ataxia. Some survive into adulthood, but seizures are refractory, and cognitive outcome is poor (Jansen FE et al. Neurology 2006;67:2224-6; cited by authors). In the current report, infantile.epileptic encephalopathies with SCN1A mutations now include, in addition to SMEI and SMEB, several infantile onset epileptic syndromes, previously thought to be cryptogenic.

\title{
EPIDEMIOLOGY AND OUTCOME OF CONVULSIVE STATUS EPILEPTICUS
}

The results of the North London Convulsive Status Epilepticus in Childhood Surveillance Study (NLCSESS) (Chin RF et al. Lancet 2006;368:222-229) are used to develop a new treatment protocol, by researchers at the Institute of Child Health, and Great Ormond Street Hospital for Children, London, UK. Status epilepticus is defined as a seizure or series of seizures that last for $30 \mathrm{~min}$ or more without regaining consciousness between seizures (ILAE Guidelines for epidemiologic studies on epilepsy. Epilepsia 1993;34:592596). A definition used for treatment purposes includes continuous seizure activity lasting 5 min, and is termed potential status epilepticus. The study was for 2 years up to the $16^{\text {th }}$ birthday and neonates were excluded. The incidence of CSE in this population was 17$23 / 100,000$ children per year. Prolonged febrile seizures were the most common cause, occurring in $32 \%$ of the total of 304 episodes of CSE. Etiology was acute symptomatic in $17 \%$, remote symptomatic in $16 \%$, previous epilepsy in $12 \%$, and unknown in $7 \%$. Age incidence was mainly 0-4 years. Incidence was increased in Asian (Indian and Pakistani) ethnic groups, and with poor socioeconomic status. Acute bacterial and virus CNS infections accounted for $19 \%$ of CSE with fever. Seizures were focal in onset in $36 \%$, becoming generalized in $95 \%$; $60 \%$ lasted longer than 60 min. CSE recurred in $17 \%$ in 1 year. Mortality of $3.4 \%$ was related to the cause (bacterial meningitis, metabolic or neurodegenerative disorder).

Analysis of data extracted from the NLCSESS population-based study supported the use of iv lorazepam as first-line treatment of CSE in preference to rectal diazepam, and iv phenytoin as second-line treatment. Prehospital treatment is important, but doses of benzodiazepine should be limited to 2 , to avoid respiratory depression. Management of an underlying acute symptomatic cause is as important as stopping the seizure in terms of longterm outcome. Treatment for possible bacterial meningitis should begin early in a child with febrile CSE and while the child is investigated. (Neville BGR, Chin RFM, Scott RC. Childhood convulsive status epilepticus: epidemiology, management and outcome. Acta Neurol Scand April 2007;115 (s186):21-24). (Respond: Dr Brian Neville, The Wolfson Centre, Mecklenburgh Square, London WC1N 2AP, UK).

COMMENT. This article was presented at an International Symposium on Status Epilepticus in Infants and Young Children in Japan, April 2006. Other papers presented and published in this supplement include "Treatment of convulsive status epilepticus (CSE)" by Sugai K, and "Hippocampal volumes and diffusion-weighted image findings in children with prolonged febrile seizures" by Natsume J et al. Sugai lists the causes of CSE in his tertiary hospital as intractable epilepsies (34-49\%), CNS infections (14-28\%), febrile seizures (13$23 \%)$, metabolic disorders (3-5\%), and cerebrovascular (1-3\%). In a city hospital in Japan, 
the most frequent cause was complex febrile seizures (57\%); epilepsies accounted for $22 \%$, and CNS infections (5\%). The most commonly used treatments for CSE in Japan are diazepam, phenytoin, and barbiturates as $1^{\text {st }}, 2^{\text {nd }}$, and $3^{\text {rd }}$-line drugs. Midazolam i.v has recently become a more favorable $2^{\text {nd }}$ line drug. (Sugai K. Acta Neurol Scand 2007;115 (s186):62-70).

Natsume and colleagues findings of hippocampal pathology following prolonged febrile seizures provide further evidence of risk of mesial temporal sclerosis (MTS) and temporal lobe epilepsy (TLE) as late complications of childhood febrile seizures. Scott RC et al postulated that MTS nay develop after a lag period (Scott RC et al. Brain 2003;126:25512557; ibid Epilepsia 2006;47:1493-1498; Ped Neur Briefs Oct 2006;20:77). Neurosurgical experience has shown that adult patients with TLE associated with MTS are more likely to have a childhood history of febrile seizures than those without MTS (Falconer MA. Epilepsia 1971;12:13-31). Perhaps the "benign" nature of febrile seizures has been overemphasized. Efforts to prevent recurrence and rapidly abort febrile seizures should be given more attention, especially in patients with a first complex febrile seizure.

\section{COGNITIVE DISORDERS IN BENIGN CHILDHOOD EPILEPSY WITH CENTROTEMPORAL SPIKES (BECTS)}

Intelligence and language functions were examined in 24 children (mean age 9 yrs; range 7-12 yrs) with BECTS and compared with a group of 16 controls matched for age and schooling, in a study at the Instituto Nazionale Neurologico, Milan, Italy. Tests measuring phonemic fluency, verbal expression of semantic knowledge, and reading comprehension revealed mild language defects. Neuropsychological functions were impaired by excess interictal EEG discharges while awake, multifocal spike location, and temporal location. A side-specific impairment was noted, with deficiencies in phonemic fluency correlating with left-sided spikes, and impaired lexical comprehension with right-sided spikes. (Riva D, Vago $\mathrm{C}$, Franceschetti $\mathrm{S}$ et al. Intellectual and language findings and their relationship to EEG characteristics in benign childhood epilepsy with centrotemporal spikes. Epilepsy Behav March 2007;10:278-285). (Respond: Dr Daria Riva, Developmental Neurology Division, Instituto Nazionale Neurologico C Besta, Via Celoria 11, Milan, Italy).

COMMENT. Children with BECTS have a slightly lower IQ and subtle language impairments compared to controls. A high spike frequency in the waking state has negative effects on cognitive and language function, whereas the sleeping spike rate shows no correlation. In this study, the BECTS-associated subtle neuropsychological dysfunction is not reflected in any school difficulties. Findings at reexamination in later childhood, when BECTS has resolved, will be of interest.

\section{ENDOCRINE EFFECTS OF VALPROATE IN ADOLESCENT GIRLS WITH EPILEPSY}

The effects of epilepsy and/or valproate (VPA) monotherapy on physical growth, weight gain, pubertal development, and hormonal status of 68 consecutive female patients aged 6-20 years (20 premenarche, 60 postmenarche) were studied at the Institute for 